\title{
Differential Diagnosis Tool for Parkinsonian Syndrome Using Multiple Structural Brain Measures
}

\author{
Miho Ota, ${ }^{1}$ Yasuhiro Nakata, ${ }^{2}$ Kimiteru Ito, ${ }^{2}$ Kouhei Kamiya, ${ }^{2}$ Masafumi Ogawa, \\ Miho Murata, ${ }^{3}$ Satoko Obu, ${ }^{1}$ Hiroshi Kunugi, ${ }^{1}$ and Noriko Sato ${ }^{2}$ \\ ${ }^{1}$ Department of Mental Disorder Research, National Institute of Neuroscience, National Center of Neurology and Psychiatry, \\ 4-1-1 Ogawa-Higashi, Kodaira, Tokyo 187-8502, Japan \\ ${ }^{2}$ Department of Radiology, National Center of Neurology and Psychiatry Hospital, 4-1-1, Ogawa-Higashi, Kodaira, \\ Tokyo 187-8551, Japan \\ ${ }^{3}$ Department of Neurology, National Center of Neurology and Psychiatry Hospital, 4-1-1 Ogawa-Higashi, Kodaira, \\ Tokyo 187-8551, Japan \\ Correspondence should be addressed to Miho Ota; ota@ncnp.go.jp
}

Received 28 November 2012; Revised 18 February 2013; Accepted 18 February 2013

Academic Editor: Wenxiang Cong

Copyright (C) 2013 Miho Ota et al. This is an open access article distributed under the Creative Commons Attribution License, which permits unrestricted use, distribution, and reproduction in any medium, provided the original work is properly cited.

\begin{abstract}
Clinical differentiation of parkinsonian syndromes such as the Parkinson variant of multiple system atrophy (MSA-P) and cerebellar subtype (MSA-C) from Parkinson's disease is difficult in the early stage of the disease. To identify the correlative pattern of brain changes for differentiating parkinsonian syndromes, we applied discriminant analysis techniques by magnetic resonance imaging (MRI). T1-weighted volume data and diffusion tensor images were obtained by MRI in eighteen patients with MSA-C, 12 patients with MSA-P, 21 patients with Parkinson's disease, and 21 healthy controls. They were evaluated using voxel-based morphometry and tract-based spatial statistics, respectively. Discriminant functions derived by step wise methods resulted in correct classification rates of 0.89 . When differentiating these diseases with the use of three independent variables together, the correct classification rate was the same as that obtained with step wise methods. These findings support the view that each parkinsonian syndrome has structural deviations in multiple brain areas and that a combination of structural brain measures can help to distinguish parkinsonian syndromes.
\end{abstract}

\section{Introduction}

Multiple system atrophy (MSA) is an adult-onset, sporadic, progressive neurodegenerative disease characterized by varying severity of parkinsonian features, and cerebellar ataxia, autonomic failure, and corticospinal disorders [1-4]. According to the clinical presentation, a parkinsonian type (MSA-P) and a cerebellar type of MSA (MSA-C) are distinguished [2] Parkinson's disease (PD) is a progressive neurodegenerative movement disorder characterized by rigidity, tremor, and bradykinesia. Its prevalence increases with age, and it affects $1 \%$ of the population over age 65 [5].

PD and MSA are both alpha-synucleinopathies $[6,7]$. Pathologically, in Parkinson's disease a massive loss of dopaminergic neurons in pars compacta of substantia nigra and intraneuronal Lewy bodies are present [8]. In MSA, neuronal loss and gliosis occur in the inferior olives, pons, transverse pontocerebellar fibers, cerebellum, substantia nigra, locus caeruleus, striatum, and the intermediolateral column of the spinal cord [9]. In MSA-P, the nigrostriatal system is the main site of pathology, but less severe degeneration can be widespread and usually includes the olivopontocerebellar system $[9,10]$. In MSA-C, the olivopontocerebellar system is mainly affected along with loss of pontine neurons and transverse pontocerebellar fibres and atrophy of the middle cerebellar peduncles (MCPs) $[9,10]$. Conventional magnetic resonance imaging (MRI) may also help distinguish the two forms of MSA. MSA-P shows "slit-like" marginal hyperintensity of the putamen [11]. Additionally, the "hot-cross bun" sign on T2-weighted and proton density images in the ventral pons has been reported to be related to MSA-C [12]. However, these MRI changes do not always occur 
[3]. Clinical differential diagnosis between PD and MSA is difficult in the early stage of the disease. Relevant works on the other tools like SPECT [13], transcranial brain sonography [14-16] and on optical coherence tomography [17] showed the effectiveness for differentiating PD from healthy volunteer. Additionally, combined use of 123I-(S)-2hydroxy-3-iodo-6-methoxy-N-((1-ethyl-2-pyrrodinyl)-methyl) benzamide (IBZM), 123I-N-v-fluoropropyl-2b-carbomethoxy-3b-(4-iodophenyl)nortropan (FP-CIT), and meta123I-iodobenzylguanidine (MIBG) distinguishes Parkinsons disease from atypical parkinsonian disorder, such as PSP and MSA with the accuracy of about $90 \%$ [18]. However, 3 SPECT/scintigraphy tests only for diagnosis are not practical.

Over the last few years, a number of MRI studies have focused on the identification of diagnostic markers helpful in the differential diagnosis of parkinsonian syndromes such as MSA, PD, and progressive supranuclear palsy (PSP) [19-22]. However, no studies have discriminated among PD, MSA-P, MSA-C, and healthy subjects simultaneously. In the present study, we hypothesized that we would be able to distinguish the PD and healthy subjects from the MSA subjects by using the infratentorial brain images and MSA-P and PD from the MSA-C and healthy subjects by using supratentorial images. The characteristic distribution of regional brain changes revealed by the gray matter volume data using the optimized, voxel-based morphometry (VBM) method and by the diffusion tensor imaging data using tract-based spatial statistics (TBSS) would have diagnostic values for discriminating such diseases.

\section{Materials and Methods}

2.1. Subjects. From November 2006 to November 2010, 200 consecutive patients whose chief complaints were parkinsonism underwent brain MRI at our institution. We excluded the patients with cerebrovascular diseases cortical infarctions, multiple lacunar lesions, leukoaraiosis, and other lesions above Fazekas's Grade 2 on T2-weighted images or fluidattenuated inversion recovery (FLAIR) MRI [23], PSP, and corticobasal degeneration (CBD). Clinical diagnosis of PD and MSA was made according to the established consensus criteria $[2,24]$. A probable clinical diagnosis was determined by two neurologist with more than 20 years of experience in the diagnosis of movement disorders (MO, MM). As a consequence, 18 consecutive patients with MSA-C, 12 patients with MSA-P, and 21 patients with PD were studied. Their characteristics are shown in Table 1. 24 out of 30 MSA patients were hospitalized for the detailed diagnosis, and the diagnosis of 30 MSA patients weas not changed during follow-up clinical assessments (mean period $=2.2$ years). As for PD, the follow-up clinical assessments were conducted (mean period $=4.7$ years) after the MRI imaging, and no additional pathology was detected. 21 age- and sex-matched healthy persons who demonstrated no current or past history of psychiatric illness or contact with psychiatric services were enrolled as controls. Participants were excluded if they had a prior medical history of central nervous system disease or severe head injury. The study protocol was approved by the
TABLE 1: Characteristics of the participants.

\begin{tabular}{lcccc}
\hline & MSA-C & MSA-P & PD & $\begin{array}{c}\text { Normal } \\
\text { volunteers }\end{array}$ \\
\hline $\begin{array}{l}\text { Mean age (years) } \\
\text { Sex (male: female) }\end{array}$ & $63.6 \pm 7.8$ & $61.9 \pm 7.7$ & $62.2 \pm 7.0$ & $62.3 \pm 5.6$ \\
$\begin{array}{l}\text { Duration of illness } \\
\text { (year) }\end{array}$ & $3.9 \pm 2.5$ & $3.3 \pm 2.6$ & $6.8 \pm 4.1$ & $10: 11$ \\
\hline
\end{tabular}

MSA-C: cerebellar form of multiple system atrophy; MSA-P: parkinsonism forms of multiple system atrophy; PD: Parkinson's disease.

ethics committee of the National Center of Neurology and Psychiatry, Japan.

2.2. MRI Data Acquisition and Processing. MR studies were performed on a Magnetom Symphony 1.5 Tesla (Siemens, Erlangen, Germany). First, high-spatial-resolution, 3-dimensional (3D) T1-weighted images of the brain were obtained for morphometric study. The 3D T1-weighted images were scanned in the sagittal plane (TE/TR: 2.64/1580 ms; flip angle: $15^{\circ}$; effective slice thickness: $1.23 \mathrm{~mm}$; slab thickness: $177 \mathrm{~mm}$; matrix: $208 \times 256$; FOV: $256 \times 315 \mathrm{~mm}^{2}$; acquisitions: 1 ) yielding 144 contiguous slices through the head. The raw $3 \mathrm{D}$ T1-weighted volume data were transferred to a workstation, and structural images were analyzed using an optimized VBM technique. Data were analyzed using Statistical Parametric Mapping 5 (SPM5) software (Welcome Department of Imaging Neuroscience, London, UK) running on MATLAB 7.0 (Math Works, Natick, MA). Images were processed using an optimized VBM script. The details of this process are described elsewhere [25]. First, each individual 3D-T1 image was normalized with the optimized VBM method. Normalized segmented images were modulated by multiplication with Jacobian determinants of the spatial normalization function to encode the deformation field for each subject, as tissue density changes in normal space. Gray matter volume and cerebrospinal fluid (CSF) volume images were smoothed using a $12 \mathrm{~mm}$ full width at half maximum of an isotropic Gaussian kernel. Diffusion tensor imaging (DTI) was then performed in the axial plane (TE/TR: 106/11,200 ms; FOV: $240 \times 240 \mathrm{~mm}^{2}$; matrix: $96 \times 96 ; 75$ continuous transverse slices; slice thickness $2.5 \mathrm{~mm}$ with no interslice gap). Diffusion was measured along 64 noncollinear directions with the use of a diffusion-weighted factor $b$ in each direction for $1000 \mathrm{~s} / \mathrm{mm}^{2}$, and one image was acquired without use of a diffusion gradient. Recently, a novel processing technique has been published. In this technique, instead of trying to match each and every voxel in different subjects, DTI data is projected on a common pseudoanatomical skeleton and therefore does not need smoothing [26]. TBSS is available as part of the FSL 4.1 software package [27]. The TBSS script runs the nonlinear registration, aligning all fractional anisotropy (FA) images to the FMRIB58_FA template, which is supplied with FSL. The script then takes the target and affine-aligns it into a $1 \times 1 \times 1 \mathrm{~mm}$ MNI152 space. Once this is done, each subject's FA image has the nonlinear transform to the target and then the affine transform to the MNI152 space applied, resulting in a transformation of the original FA image into 
the MNI152 space. Next, TBSS creates the mean of all aligned FA images and applies thinning of the local tract structure to create a skeletonized mean FA image. In order to exclude areas of low FA and/or high intersubject variability from the statistical analysis, TBSS thresholds a mean FA skeleton with a certain FA value, typically 0.2 . The resulting binary skeleton mask is a pseudoanatomical representation of the main fiber tracks and defines the set of voxels used in all subsequent processing. Finally, TBSS projects each subject's aligned FA image onto the skeleton. This results in skeletonized FA data. It is this file that feeds into the voxelwise statistics. In addition to DTI and 3D T1-weighted images, conventional axial T2weighted images (TE/TR: 95/3500 ms; flip angle: $150^{\circ}$; slice thickness: $5 \mathrm{~mm}$; intersection gap: $1.75 \mathrm{~mm}$; matrix: $448 \times$ 512; field of view (FOV): $210 \times 240 \mathrm{~mm}^{2}$; acquisitions: 1 ) and fluid attenuation inversion recovery images in the axial plane (TE/TR: $101 / 8800 \mathrm{~ms}$; flip angle: $150^{\circ}$; slice thickness: $3 \mathrm{~mm}$; intersection gap: $1.75 \mathrm{~mm}$; matrix: $448 \times 512$; FOV: $210 \times 240 \mathrm{~mm}^{2}$; acquisition: 1) were acquired to exclude cerebrovascular disease or other diseases such as tumors, and hydrocephalus. On conventional MRI, no abnormal findings were detected in the brain of any subject.

2.3. Statistical Analysis. We first evaluated the differences between the patients and healthy subjects using analysis of variance (ANOVA). These tests were performed with the SPSS software ver. 11 (SPSS Japan, Tokyo, Japan). There were no significant differences in age among patients and controls, but there were statistically significant differences in duration of illness between the patients with MSA-P and with PD $(P=$ $0.012)$ and with MSA-C and with PD $(P=0.005)$.

The discriminant function analyses were then conducted to assess the ability of a combination of brain anatomical variables to distinguish between patients with MSA-C, MSA-P, Parkinson's disease, and controls. The independent variables were the volume data and fractional anisotropy value derived from the normalized individual image using the region of interests (ROI) method. ROIs were put on the "single_subj_T1.nii" image regarded as the anatomically standard image in SPM5, in the fourth ventricle, cerebellum hemisphere; these were derived from the WFU_PickAtlas, extension program of SPM5 [28, 29]. We also put ROIs on the "FMRIB58_FA-skeleton_1 mm.nii" image, which is the anatomically standard image in FSL, in the MCP, superior cerebellar peduncle (SCP), pons, substantia nigra, superior temporal white matter region, prefrontal white matter regions, and primary motor region where previous studies showed differences among the patients with MSA-C, MSAP, PD, and controls (Figure 1) [20, 22, 30-37]. The value of a particular tissue was extracted using the software MarsBar [38], an extension program of SPM5.

The Box's $M$ test confirmed the inequality of the group covariance matrices (Box- $M=76.63 ; P<0.001$ ). Discriminant functions were derived by step wise methods based on Mahalanobis' distance. The step wise selection criteria were decided by the overall multivariate $F$ value of each variable to test differences between the patients and controls and to maximize the discriminant function between the groups. At
TABLE 2: Mean fractional anisotropy value in each region of interests and 4 th ventricular and cerebellar volumes in patients with MSA-C, MSA-P, PD, and controls.

\begin{tabular}{lcccc}
\hline & MSA-C & MSA-P & PD & Normal \\
\hline 4th Vent_vol & $0.31 \pm 0.07$ & $0.28 \pm 0.07$ & $0.19 \pm 0.04$ & $0.20 \pm 0.04$ \\
Cerebellum_vol & $0.28 \pm 0.04$ & $0.30 \pm 0.03$ & $0.33 \pm 0.04$ & $0.34 \pm 0.04$ \\
MCP & $0.32 \pm 0.05$ & $0.37 \pm 0.05$ & $0.41 \pm 0.03$ & $0.39 \pm 0.03$ \\
SCP & $0.44 \pm 0.02$ & $0.43 \pm 0.02$ & $0.45 \pm 0.04$ & $0.47 \pm 0.03$ \\
Pons & $0.12 \pm 0.01$ & $0.13 \pm 0.01$ & $0.14 \pm 0.01$ & $0.13 \pm 0.01$ \\
SN & $0.53 \pm 0.04$ & $0.54 \pm 0.01$ & $0.54 \pm 0.05$ & $0.56 \pm 0.04$ \\
ST & $0.21 \pm 0.01$ & $0.13 \pm 0.01$ & $0.13 \pm 0.01$ & $0.21 \pm 0.02$ \\
PF & $0.05 \pm 0.00$ & $0.06 \pm 0.01$ & $0.06 \pm 0.00$ & $0.05 \pm 0.01$ \\
PM & $0.20 \pm 0.02$ & $0.21 \pm 0.02$ & $0.22 \pm 0.02$ & $0.21 \pm 0.02$ \\
\hline
\end{tabular}

4th Vent_vol: fourth ventricle volume; cerebellum_vol: cerebellum volume; MSA-C: cerebellar form of multiple system atrophy; MSA-P: parkinsonism forms of multiple system atrophy; MCP: middle cerebellar peduncle; SCP: superior cerebellar peduncle; PD: Parkinson's disease; SN: substantia nigra; ST: superior temporal region; PF: prefrontal region; PM: primary motor region.

TABLE 3: The coefficients of discriminant analysis.

\begin{tabular}{lcccc}
\hline & & Factor 1 & Factor 2 & Factor 3 \\
\hline \multirow{4}{*}{ Stepwise method } & 4th Vent_vol & 1.74 & 19.47 & 3.33 \\
& SN & 19.16 & -9.81 & 38.32 \\
& ST & -127.87 & 3.14 & -32.07 \\
& PF & 196.3 & 80.22 & -188.97 \\
& (Constant) & 0.66 & -4.13 & -5.99 \\
\hline \multirow{2}{*}{ Two independent } & Pons & -76.33 & 69.41 & $(-)$ \\
variables analysis & ST & 97.92 & 13.01 & $(-)$ \\
& (Constant) & -6.98 & 11.27 & $(-)$ \\
\hline \multirow{3}{*}{ Three independent } & 4th Vent_vol & 1.14 & 15.82 & 12.23 \\
variables analysis & MCP & -24.59 & -5.39 & 23.04 \\
& ST & 100.68 & -13.21 & 6.19 \\
& (Constant) & -4.01 & -3.68 & -13.58 \\
\hline
\end{tabular}

4th Vent_vol: fourth ventricle volume; MCP: middle cerebellar peduncle; SN: substantia nigra; ST: superior temporal region; PM: primary motor region.

the same time, we entered the two or three independent variables together and estimated the predictive power of the discriminant function.

\section{Results}

We first calculated the volume and FA value from the spatially normalized images using ROIs. The mean values of these parameters are summarized in Table 2 and Figure 2. The mean FA value of the prefrontal region was too small to examine, so we did not evaluate the influence of the FA value in the prefrontal white matter region. We then conducted discriminant function analyses. The following five variables were entered in a step wise manner: fourth ventricle volume, substantia nigra, superior temporal, and prefrontal white matter region. The discriminant coefficients are shown in Table 3. The use of these variables resulted in correct 
TABLE 4: Classification results.

\begin{tabular}{|c|c|c|c|c|c|c|c|}
\hline & & & \multicolumn{5}{|c|}{ Predicted group membership } \\
\hline & & & MSA-C & MSA-P & $\mathrm{PD}$ & Control & Total \\
\hline \multicolumn{8}{|c|}{ Stepwise method ( $88.9 \%$ of original grouped cases correctly classified) } \\
\hline \multirow{8}{*}{ Original data } & \multirow{3}{*}{ Count } & MSA-C & 15 & 0 & 0 & 3 & 18 \\
\hline & & MSA-P & 0 & 9 & 3 & 0 & 12 \\
\hline & & $\mathrm{PD}$ & 0 & 2 & 19 & 0 & 21 \\
\hline & \multirow{5}{*}{$\%$} & Control & 0 & 0 & 0 & 21 & 21 \\
\hline & & MSA-C & 83.3 & 0 & 0 & 16.7 & 100 \\
\hline & & MSA-P & 0 & 75.0 & 25.0 & 0 & 100 \\
\hline & & $\mathrm{PD}$ & 0 & 9.5 & 90.5 & 0 & 100 \\
\hline & & Control & 0.0 & 0 & 0 & 100.0 & 100 \\
\hline \multicolumn{8}{|c|}{ Two independent variables ( $84.7 \%$ of original grouped cases correctly classified) } \\
\hline \multirow{8}{*}{ Original data } & \multirow{4}{*}{ Count } & MSA-C & 15 & 0 & 0 & 3 & 18 \\
\hline & & MSA-P & 0 & 10 & 2 & 0 & 12 \\
\hline & & PD & 0 & 4 & 17 & 0 & 21 \\
\hline & & Control & 1 & 1 & 0 & 19 & 21 \\
\hline & \multirow{4}{*}{$\%$} & MSA-C & 83.3 & 0.0 & 0 & 16.7 & 100 \\
\hline & & MSA-P & 0 & 83.3 & 16.7 & 0 & 100 \\
\hline & & $\mathrm{PD}$ & 0 & 19.0 & 81.0 & 0 & 100 \\
\hline & & Control & 4.8 & 4.8 & 0.0 & 90.5 & 100 \\
\hline \multicolumn{8}{|c|}{ Three independent variables ( $88.9 \%$ of original grouped cases correctly classified) } \\
\hline \multirow{8}{*}{ Original data } & \multirow{4}{*}{ Count } & MSA-C & 14 & 0 & 0 & 4 & 18 \\
\hline & & MSA-P & 0 & 10 & 2 & 0 & 12 \\
\hline & & $\mathrm{PD}$ & 0 & 1 & 20 & 0 & 21 \\
\hline & & Control & 0 & 1 & 0 & 20 & 21 \\
\hline & \multirow{4}{*}{$\%$} & MSA-C & 77.8 & 0.0 & 0 & 22.2 & 100 \\
\hline & & MSA-P & 0 & 83.3 & 16.7 & 0 & 100 \\
\hline & & $\mathrm{PD}$ & 0 & 4.8 & 95.2 & 0 & 100 \\
\hline & & Control & 0.0 & 4.8 & 0.0 & 95.2 & 100 \\
\hline
\end{tabular}

MSA-C: cerebellar form of multiple system atrophy; MSA-P: parkinsonism forms of multiple system atrophy; PD: Parkinson's disease.

classification rates of $0.89\left(\chi^{2}=294.66 ; \mathrm{df}=12 ; P<0.001\right.$; Wilks' lambda $=0.012)($ Table 4$)$.

The correct classification rates of each combination used to run the discriminant function analyses using two or three independent variables together are listed in Table 5. The highest correct classification rates were measured when we estimated the FA value of the "pons and superior temporal region" and "superior temporal region, MCP and fourth ventricle volume", respectively. Table 3 shows the discriminant coefficients, and Table 4 shows the correct classification rates derived from the analyses using two or three independent variables together, in the same way. Figure 3 shows the discriminant scores of each subject, calculated by the analysis using three independent variables together.

\section{Discussion}

We found that the step wise discriminant function analysis identified with fairly good accuracy the combinations of ROIs that characterized brain anatomical features distinguishing the patients with MSA-C, MSA-P, PD, and healthy subjects, and that when discriminate analysis was conducted using the fourth ventricle volume and the FA value of MCP and superior temporal region as independent variables together, the correct classification rate was the same as that of step wise discriminant function analysis.

One study showed that patients with MSA-C and MSA$\mathrm{P}$ share similar diffusion tensor imaging features in the infratentorial region [22]. Furthermore, the combination of DTI metrics can be used to distinguish between patients with MSA and with PD. However, they could not differentiate the patients with PD from healthy subjects. This may be because they were focused on the infratentorial FA value and did not investigate the focal lesions related to parkinsonism. In this study, we used the FA value of the superior temporal regions, known to be impaired in PD as an independent variable, so we could discriminate the patients with $\mathrm{PD}$ from healthy subjects and with MSA-C from those with MSA-P [30-32, 34, 35].

One study reported discriminating patients with MSAP, PD, PSP, and healthy subjects [20]. They indicated that investigating the degeneration of the MCP is useful for the in vivo differential diagnosis of MSA-P and PD. These 
TABLE 5: The correct classification rates of each combination of independent variables.

(a) Two independent variables

\begin{tabular}{|c|c|c|c|c|c|c|c|c|}
\hline & 4th Vent_vol & Cerebellum_vol & MCP & SCP & Pons & SN & ST & PM \\
\hline 4th Vent_vol & & 0.514 & 0.583 & 0.611 & 0.583 & 0.556 & 0.819 & 0.431 \\
\hline Cerebellum_vol & & & 0.528 & 0.556 & 0.514 & 0.458 & 0.722 & 0.458 \\
\hline MCP & & & & 0.708 & 0.472 & 0.667 & 0.833 & 0.528 \\
\hline SCP & & & & & 0.681 & 0.528 & 0.736 & 0.542 \\
\hline Pons & & & & & & 0.667 & 0.847 & 0.514 \\
\hline SN & & & & & & & 0.722 & 0.583 \\
\hline ST & & & & & & & & 0.653 \\
\hline PM & & & & & & & & \\
\hline
\end{tabular}

4th Vent_vol: fourth ventricle volume; cerebellum_vol: cerebellum volume; MCP: middle cerebellar peduncle; SCP: superior cerebellar peduncle; SN: substantia nigra; ST: superior temporal region; PM: primary motor region.

(b) Three independent variables

\begin{tabular}{|c|c|c|c|c|c|c|c|c|}
\hline & & & & & & & & Accuracy \\
\hline 4th Vent_vol & Cerebellum_vol & MCP & & & & & & 0.556 \\
\hline 4th Vent_vol & Cerebellum_vol & & SCP & & & & & 0.681 \\
\hline 4th Vent_vol & Cerebellum_vol & & & Pons & & & & 0.569 \\
\hline 4th Vent_vol & Cerebellum_vol & & & & $\mathrm{SN}$ & & & 0.583 \\
\hline 4th Vent_vol & Cerebellum_vol & & & & & ST & & 0.861 \\
\hline 4th Vent_vol & Cerebellum_vol & & & & & & PM & 0.583 \\
\hline 4th Vent_vol & & MCP & SCP & & & & & 0.708 \\
\hline 4th Vent_vol & & MCP & & Pons & & & & 0.542 \\
\hline 4th Vent_vol & & MCP & & & $\mathrm{SN}$ & & & 0.653 \\
\hline 4th Vent_vol & & MCP & & & & ST & & 0.889 \\
\hline 4th Vent_vol & & MCP & & & & & $\mathrm{PM}$ & 0.569 \\
\hline 4th Vent_vol & & & SCP & Pons & & & & 0.722 \\
\hline 4th Vent_vol & & & SCP & & $\mathrm{SN}$ & & & 0.625 \\
\hline 4th Vent_vol & & & SCP & & & ST & & 0.833 \\
\hline 4th Vent_vol & & & SCP & & & & PM & 0.681 \\
\hline 4th Vent_vol & & & & Pons & SN & & & 0.681 \\
\hline 4th Vent_vol & & & & Pons & & ST & & 0.889 \\
\hline 4th Vent_vol & & & & Pons & & & $\mathrm{PM}$ & 0.597 \\
\hline 4th Vent_vol & & & & & SN & ST & & 0.875 \\
\hline 4th Vent_vol & & & & & $\mathrm{SN}$ & & PM & 0.611 \\
\hline \multirow[t]{16}{*}{ 4th Vent_vol } & & & & & & ST & $\mathrm{PM}$ & 0.861 \\
\hline & Cerebellum_vol & $\mathrm{MCP}$ & SCP & & & & & 0.681 \\
\hline & Cerebellum_vol & MCP & & Pons & & & & 0.486 \\
\hline & Cerebellum_vol & MCP & & & SN & & & 0.639 \\
\hline & Cerebellum_vol & $\mathrm{MCP}$ & & & & ST & & 0.833 \\
\hline & Cerebellum_vol & MCP & & & & & $\mathrm{PM}$ & 0.528 \\
\hline & Cerebellum_vol & & SCP & Pons & & & & 0.625 \\
\hline & Cerebellum_vol & & SCP & & $\mathrm{SN}$ & & & 0.583 \\
\hline & Cerebellum_vol & & SCP & & & ST & & 0.764 \\
\hline & Cerebellum_vol & & SCP & & & & PM & 0.583 \\
\hline & Cerebellum_vol & & & Pons & $\mathrm{SN}$ & & & 0.653 \\
\hline & Cerebellum_vol & & & Pons & & ST & & 0.833 \\
\hline & Cerebellum_vol & & & Pons & & & $\mathrm{PM}$ & 0.528 \\
\hline & Cerebellum_vol & & & & $\mathrm{SN}$ & ST & & 0.792 \\
\hline & Cerebellum_vol & & & & $\mathrm{SN}$ & & $\mathrm{PM}$ & 0.583 \\
\hline & Cerebellum_vol & & & & & ST & $\mathrm{PM}$ & 0.792 \\
\hline
\end{tabular}


(b) Continued.

\begin{tabular}{|c|c|c|c|c|c|c|}
\hline & & & & & & Accuracy \\
\hline МСР & SCP & Pons & & & & 0.681 \\
\hline MCP & SCP & & SN & & & 0.694 \\
\hline МCP & SCP & & & ST & & 0.819 \\
\hline МСР & SCP & & & & PM & 0.681 \\
\hline МСР & & Pons & SN & & & 0.694 \\
\hline МСP & & Pons & & ST & & 0.806 \\
\hline МСР & & Pons & & & PM & 0.486 \\
\hline МCP & & & SN & ST & & 0.833 \\
\hline МСР & & & SN & & PM & 0.625 \\
\hline MCP & & & & ST & PM & 0.819 \\
\hline & SCP & Pons & SN & & & 0.667 \\
\hline & SCP & Pons & & ST & & 0.819 \\
\hline & SCP & Pons & & & PM & 0.681 \\
\hline & SCP & & SN & ST & & 0.792 \\
\hline & SCP & & SN & & PM & 0.611 \\
\hline & SCP & & & ST & PM & 0.722 \\
\hline & & Pons & SN & ST & & 0.819 \\
\hline & & Pons & SN & & PM & 0.694 \\
\hline & & Pons & & ST & PM & 0.819 \\
\hline & & & SN & ST & PM & 0.764 \\
\hline
\end{tabular}

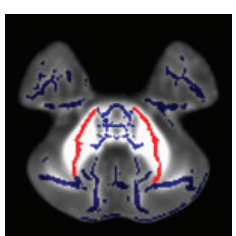

(a) Middle cerebellar peduncle

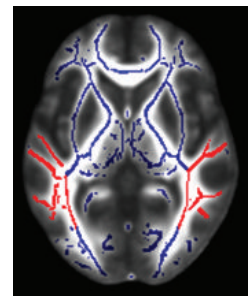

(e) Superior temporal region

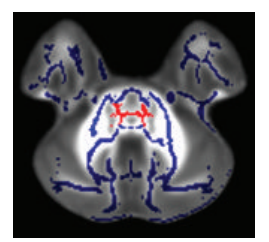

(b) Pons

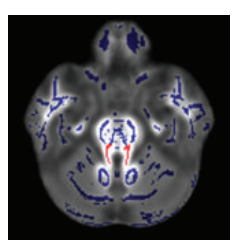

(c) Superior cerebellar peduncle

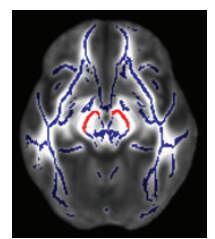

(d) Substantia nigra

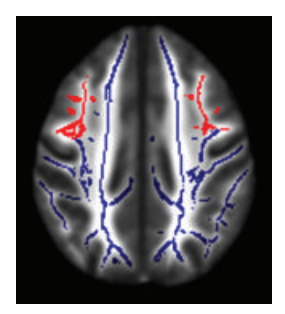

(f) Prefrontal region

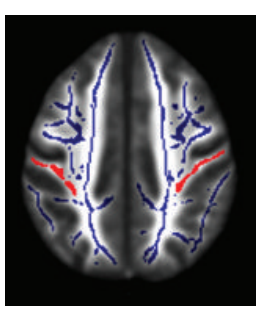

(g) Primary motor region

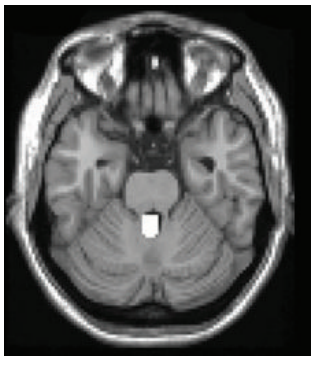

(h) 4th ventricle

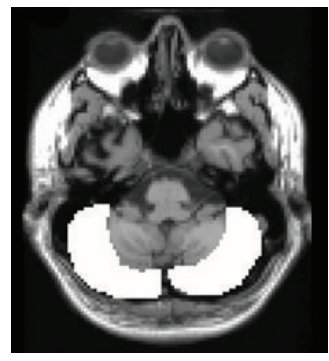

(i) Cerebellar hemisphere

FIGURE 1: Locations of regions of interest. From (a) to (g) were put on the "FMRIB58_FA-skeleton_1 mm.nii" image, the anatomically standard image in FSL. Background fractional anisotropy image was the "MNI152_T1_1 mm.nii," which was also the standard image in FSL. (h) and (i) were put on a "single_subj_T1.nii" image regarded as the anatomically standard image in SPM5. 


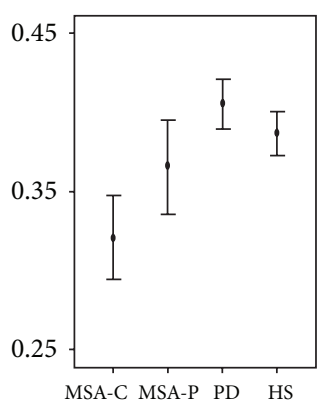

(a) Middle cerebellar peduncle

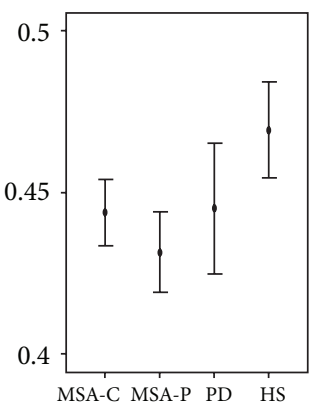

(b) Superior cerebellar peduncle

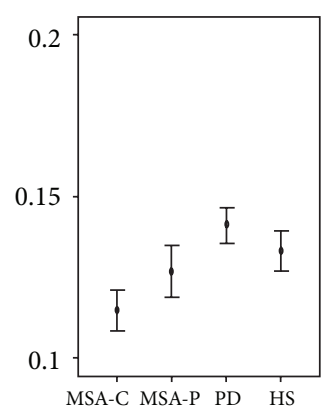

(c) Pons

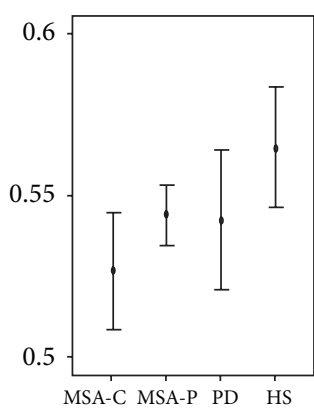

(d) Substantia nigra

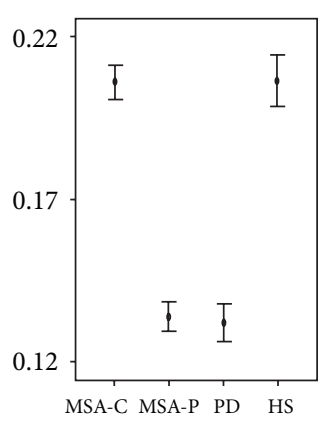

(e) Superior temporal reg-

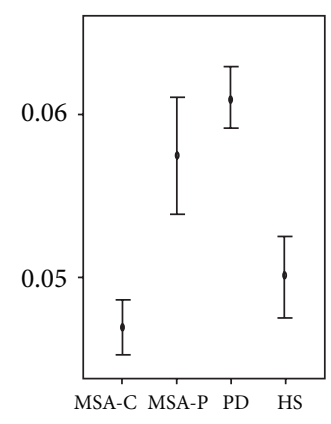

(f) Prefrontal region

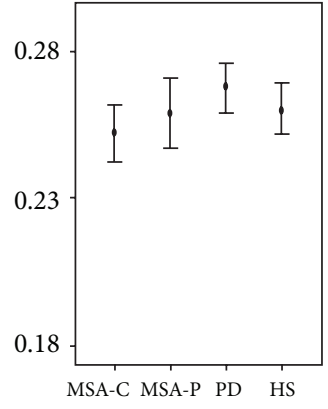

(g) Primary motor region ion

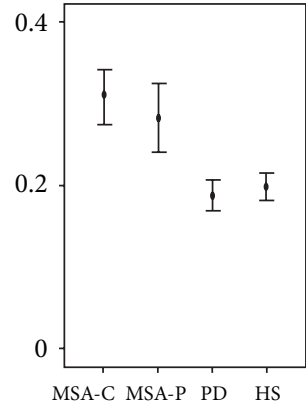

(h) 4 th ventricle

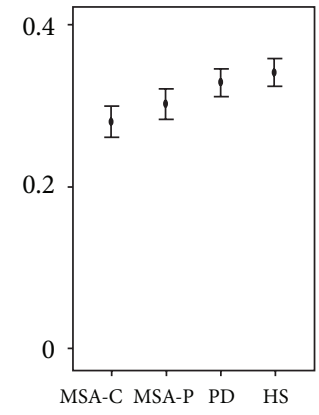

(i) Cerebellar hemisphere

Figure 2: A graphic presentation of mean fractional anisotropy values in each region and mean volumes of 4th ventricular and cerebellum. MSA-C: multiple system atrophy with predominant cerebellar ataxia; MSA-P: multiple system atrophy with predominant parkinsonism; PD: Parkinson's disease; HS: healthy subjects.

results are congruent with our study. Establishing a means of differentiation using MR imaging would have potential therapeutic implications.

In this study, the participants with PD had a statistically longer duration of illness than those with MSA. It is known that MRI studies with PD show slight or no gray matter atrophy in early- to moderate-stage patients, whereas later-stage patients exhibited marked cortical atrophy [31]. We used the FA values for the independent variables to differentiate the patients with PD from others. White matter which appears normal on conventional MRI can show FA abnormalities, possibly permitting an earlier identification of the disease process which involves white matter tracts of the brain [33]. In addition, we successfully differentiated the patients with MSA-P from those with MSA-C using the same parameter.
There is significant value to distinguishing these diseases using DTI metrics.

All voxel-based analysis methods are susceptible to the effects of the spatial normalization transformation that registers images of different individuals. Regions in which this spatial transformation has relatively lower accuracy will tend to display artificially higher variability, which will adversely affect statistical significance. To date, TBSS is considered more robust and better suited for whole brain DTI data analysis. However, there are some limitations with the TBSS analysis. First, "FMRIB58_FA-skeleton_1 mm.nii" did not cover the thalamus and striatum, both of which have significant relationships with parkinsonian features [39]. A previous study demonstrated signal changes of the MR image after levodopa administration in an anatomical cluster which included the 

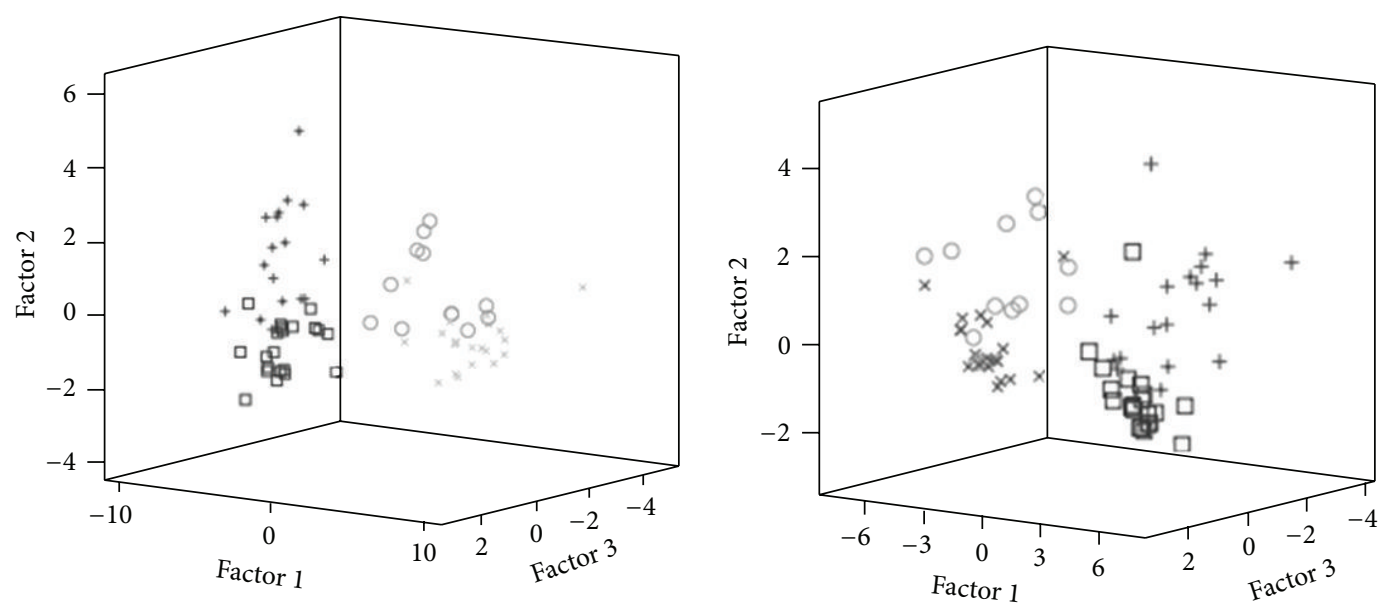

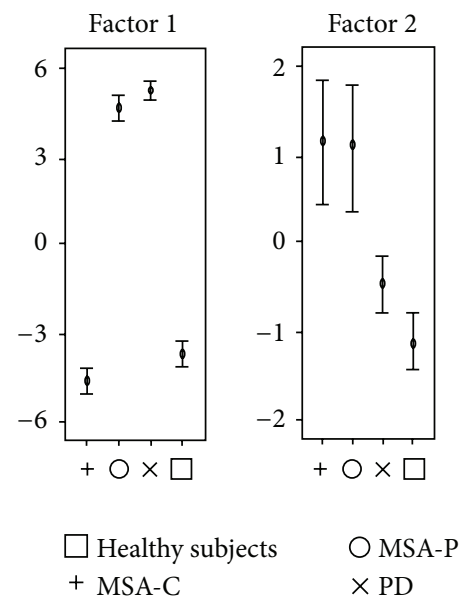

(a)
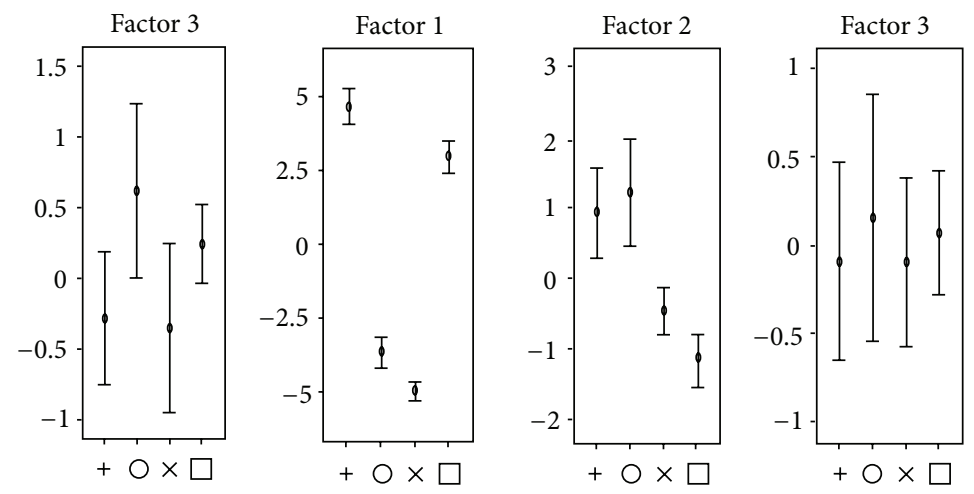

O MSA-P $\times$ PD

(b)

FIGURE 3: 3-dimensional scattered plots showed the discriminant scores of each subject. These were calculated (a) by the analysis using step wise method and (b) using three independent variables together. Factors 1-3 were defined in Table 3.

substantia nigra, tegmental ventral area subthalamic nucleus bilaterally, the principal origin, and first relay nuclei of projections in brain dopaminergic systems [40]. Therefore, we do not recommend using dopamine-rich regions such as the thalamus and striatum in discriminant function analysis for parkinsonian syndrome. Second, the predominant motor feature can change with time. The designation of MSA-P and MSA-C refers to the predominant feature at the time the patient is evaluated, and the predominant feature can change with time [2]. All of our MSA-C samples did not change the diagnosis to the MSA-P during follow-up clinical assessments; however, the discriminant method in this study would be fitted for the initial diagnosis. Third, our study is a small cross-sectional study, and we did not validate this discrimination method using another independent sample. MSA-P and MSA-C without cerebrovascular findings were so scarce, and we did not gather sufficient sample size. Further work with a large sample is required for the development of better discriminant capability and, if feasible, with data on another parkinsonism, PSP, would bring further clinical advantage.

\section{Conclusions}

Discriminant functions derived by step wise methods resulted in correct classification rates of 0.89 . The present methods for automated analysis of morphometric data largely support findings from earlier studies using expert-guided ROIs or automated procedures. These findings support the view that each parkinsonian syndrome has structural deviations in multiple brain areas, and discriminant function analysis in this paper may provide objective biological information adjunct to the clinical diagnosis of parkinsonian syndromes.

\section{References}

[1] F. Geser, G. K. Wenning, K. Seppi et al., "Progression of multiple system atrophy (MSA): a prospective natural history study by the European MSA Study Group (EMSA SG)," Movement Disorders, vol. 21, no. 2, pp. 179-186, 2006.

[2] S. Gilman, G. K. Wenning, P. A. Low et al., "Second consensus statement on the diagnosis of multiple system atrophy," Neurology, vol. 71, no. 9, pp. 670-676, 2008. 
[3] N. Quinn, "Multiple system atrophy-the nature of the beast," Journal of Neurology, Neurosurgery \& Psychiatry, vol. 52, pp. 7889, 1986.

[4] G. K. Wenning, F. Tison, Y. B. Shlomo, S. E. Daniel, and N. P. Quinn, "Multiple system atrophy: a review of 203 pathologically proven cases," Movement Disorders, vol. 12, no. 2, pp. 133-147, 1997.

[5] D. Aarsland, K. Andersen, J. P. Larsen, A. Lolk, H. Nielsen, and P. Kragh-Sørensen, "Risk of dementia in Parkinson's disease: a community-based, prospective study," Neurology, vol. 56, no. 6 , pp. 730-736, 2001.

[6] M. G. Spillantini, R. A. Crowther, R. Jakes, N. J. Cairns, P. L. Lantos, and M. Goedert, "Filamentous $\alpha$-synuclein inclusions link multiple system atrophy with Parkinson's disease and dementia with Lewy bodies," Neuroscience Letters, vol. 251, no. 3, pp. 205208, 1998.

[7] K. Wakabayashi, M. Yoshimoto, S. Tsuji, and H. Takahashi, “ $\alpha$ synuclein immunoreactivity in glial cytoplasmic inclusions in multiple system atrophy," Neuroscience Letters, vol. 249, no. 2-3, pp. 180-182, 1998.

[8] J. M. Fearnley and A. J. Lees, "Ageing and Parkinson's disease: substantia nigra regional selectivity," Brain, vol. 114, part 5, pp. 2283-2301, 1991.

[9] G. K. Wenning, F. Tison, L. Elliott, N. P. Quinn, and S. E. Daniel, "Olivopontocerebellar pathology in multiple system atrophy," Movement Disorders, vol. 11, no. 2, pp. 157-162, 1996.

[10] A. Kume, A. Takahashi, and Y. Hashizume, "Neuronal cell loss of the striatonigral system in multiple system atrophy," Journal of the Neurological Sciences, vol. 117, no. 1-2, pp. 33-40, 1993.

[11] K. Bhattacharya, D. Saadia, B. Eisenkraft et al., "Brain magnetic resonance imaging in multiple-system atrophy and Parkinson disease: a diagnostic algorithm," Archives of Neurology, vol. 59, no. 5, pp. 835-842, 2002.

[12] M. Savoiardo, L. Strada, F. Girotti et al., "Olivopontocerebellar atrophy: MR diagnosis and relationship to multisystem atrophy," Radiology, vol. 174, no. 3, pp. 693-696, 1990.

[13] J. Spiegel, M. O. Möllers, W. H. Jost et al., "FP-CIT and MIBG scintigraphy in early Parkinson's disease," Movement Disorders, vol. 20, no. 5, pp. 552-561, 2005.

[14] G. Becker, J. Seufert, U. Bogdahn, H. Reichmann, and K. Reiners, "Degeneration of substantia nigra in chronic Parkinson's disease visualized by transcranial color-coded real-time sonography," Neurology, vol. 45, no. 1, pp. 182-184, 1995.

[15] K. Busse, R. Heilmann, S. Kleinschmidt et al., "Value of combined midbrain sonography, olfactory and motor function assessment in the differential diagnosis of early Parkinson's disease," Journal of Neurology, Neurosurgery \& Psychiatry, vol. 83, no. 4, pp. 441-447, 2012.

[16] M. Izawa Okawa and H. Miwa, "Transcranial sonography findings in Parkinson's disease," Brain Nerve, vol. 64, no. 4, pp. 413422, 2012.

[17] P. Albrecht, A. K. Müller, M. Südmeyer et al., "Optical coherence tomography in parkinsonian syndromes," PLOS ONE, vol. 7, Article ID e34891, 2012.

[18] M. Südmeyer, C. Antke, T. Zizek et al., "Diagnostic accuracy of combined FP-CIT, IBZM, and MIBG scintigraphy in the differential diagnosis of degenerative parkinsonism: a multidimensional statistical approach," Journal of Nuclear Medicine, vol. 52, no. 5, pp. 733-740, 2011.

[19] R. L. Gama, D. F. G. Távora, R. C. Bomfim, C. E. Silva, V. M. de Bruin, and P. F. de Bruin, "Morphometry MRI in the differential diagnosis of parkinsonian syndromes," Arquivos de Neuro-Psiquiatria, vol. 68, no. 3, pp. 333-338, 2010.

[20] G. Nicoletti, R. Lodi, F. Condino et al., "Apparent diffusion coefficient measurements of the middle cerebellar peduncle differentiate the Parkinson variant of MSA from Parkinson's disease and progressive supranuclear palsy," Brain, vol. 129, no. 10, pp. 2679-2687, 2006.

[21] A. Quattrone, G. Nicoletti, D. Messina et al., "MR imaging index for differentiation of progressive supranuclear palsy from Parkinson disease and the Parkinson variant of multiple system atrophy," Radiology, vol. 246, no. 1, pp. 214-221, 2008.

[22] M. F. H. Schocke, K. Seppi, R. Esterhammer et al., "Diffusionweighted MRI differentiates the Parkinson variant of multiple system atrophy from PD," Neurology, vol. 58, no. 4, pp. 575-580, 2002.

[23] F. Fazekas, J. B. Chawluk, and A. Alavi, "MR signal abnormalities at $1.5 \mathrm{~T}$ in Alzheimer's dementia and normal aging," American Journal of Roentgenology, vol. 149, no. 2, pp. 351-356, 1987.

[24] W. R. G. Gibb and A. J. Lees, "The relevance of the Lewy body to the pathogenesis of idiopathic Parkinson's disease," Journal of Neurology, Neurosurgery \& Psychiatry, vol. 51, pp. 745-752, 1998.

[25] C. D. Good, I. Johnsrude, J. Ashburner, R. N. A. Henson, K. J. Friston, and R. S. J. Frackowiak, "Cerebral asymmetry and the effects of sex and handedness on brain structure: a voxel-based morphometric analysis of 465 normal adult human brains," NeuroImage, vol. 14, no. 3, pp. 685-700, 2001.

[26] S. M. Smith, M. Jenkinson, H. Johansen-Berg et al., "Tractbased spatial statistics: voxelwise analysis of multi-subject diffusion data," NeuroImage, vol. 31, no. 4, pp. 1487-1505, 2006.

[27] S. M. Smith, M. Jenkinson, M. W. Woolrich et al., "Advances in functional and structural MR image analysis and implementation as FSL," NeuroImage, vol. 23, no. 1, pp. S208-S219, 2004.

[28] J. A. Maldjian, P. J. Laurienti, R. A. Kraft, and J. H. Burdette, "An automated method for neuroanatomic and cytoarchitectonic atlas-based interrogation of fMRI data sets," NeuroImage, vol. 19, no. 3, pp. 1233-1239, 2003.

[29] J. A. Maldjian, P. J. Laurienti, and J. H. Burdette, "Precentral gyrus discrepancy in electronic versions of the Talairach atlas," NeuroImage, vol. 21, no. 1, pp. 450-455, 2004.

[30] M. K. Beyer, C. C. Janvin, J. P. Larsen, and D. Aarsland, "A magnetic resonance imaging study of patients with Parkinson's disease with mild cognitive impairment and dementia using voxel-based morphometry," Journal of Neurology, Neurosurgery \& Psychiatry, vol. 78, no. 3, pp. 254-259, 2007.

[31] P. Borghammer, K. Østergaard, P. Cumming et al., "A deformation-based morphometry study of patients with early-stage Parkinson's disease," European Journal of Neurology, vol. 17, no. 2, pp. 314-320, 2010.

[32] A. Gerhard, N. Pavese, G. Hotton et al., "In vivo imaging of microglial activation with [11C](R)-PK11195 PET in idiopathic Parkinson's disease," Neurobiology of Disease, vol. 21, no. 2, pp. 404-412, 2006.

[33] N. Prakash, N. Hageman, X. Hua, A. W. Toga, S. L. Perlman, and N. Salamon, "Patterns of fractional anisotropy changes in white matter of cerebellar peduncles distinguish spinocerebellar ataxia-1 from multiple system atrophy and other ataxia syndromes," NeuroImage, vol. 47, supplement 2, pp. T72-T81, 2009.

[34] B. Ramírez-Ruiz, M. J. Martí, E. Tolosa et al., "Longitudinal evaluation of cerebral morphological changes in Parkinson's disease with and without dementia," Journal of Neurology, vol. 252, no. 11, pp. 1345-1352, 2005. 
[35] K. Specht, M. Minnerop, M. Abele, J. Reul, U. Wüllner, and T. Klockgether, "In vivo voxel-based morphometry in multiple system atrophy of the cerebellar type," Archives of Neurology, vol. 60, no. 10, pp. 1431-1435, 2003.

[36] K. Specht, M. Minnerop, J. Müller-Hübenthal, and T. Klockgether, "Voxel-based analysis of multiple-system atrophy of cerebellar type: complementary results by combining voxelbased morphometry and voxel-based relaxometry," NeuroImage, vol. 25, no. 1, pp. 287-293, 2005.

[37] L. C. Tzarouchi, L. G. Astrakas, S. Konitsiotis et al., "Voxel-based morphometry and voxel-based relaxometry in parkinsonian variant of multiple system atrophy," Journal of Neuroimaging, vol. 20, no. 3, pp. 260-266, 2010.

[38] M. Brett, J.-L. Anton, R. Valabregue, and J.-B. Poline, "Region of interest analysis using an SPM toolbox," in Proceedings of the 8th International Conference on Functional Mapping of the Human Brain, Sendai, Japan, June 2002, Available on CD-ROM in NeuroImage, vol. 16, no. 2, 2002.

[39] P. Remy, M. Doder, A. Lees, N. Turjanski, and D. Brooks, "Depression in Parkinson's disease: loss of dopamine and noradrenaline innervation in the limbic system," Brain, vol. 128, no. 6, pp. 1314-1322, 2005.

[40] P. Salgado-Pineda, P. Delaveau, C. Falcon, and O. Blin, "Brain T1 intensity changes after levodopa administration in healthy subjects: a voxel-based morphometry study," British Journal of Clinical Pharmacology, vol. 62, no. 5, pp. 546-551, 2006. 


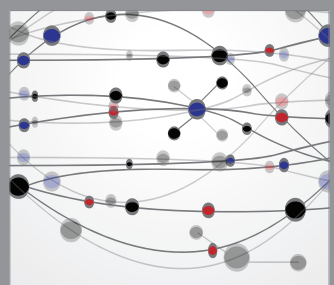

The Scientific World Journal
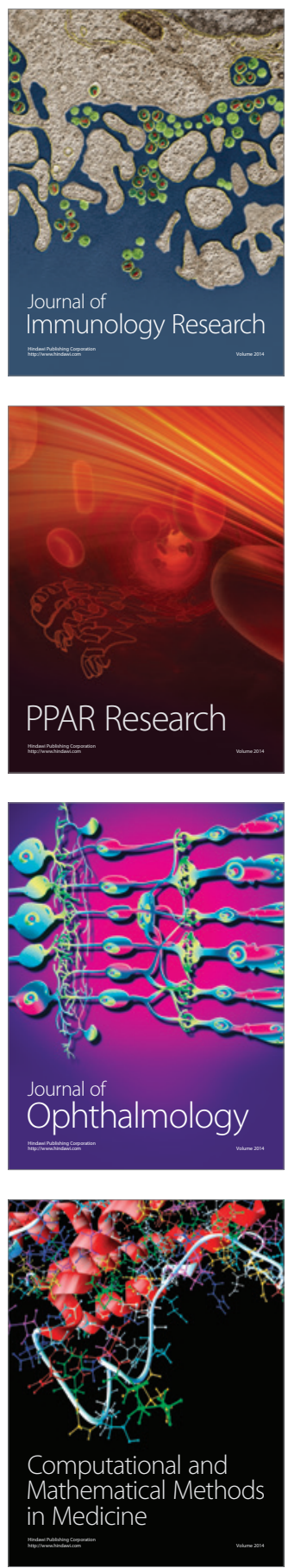

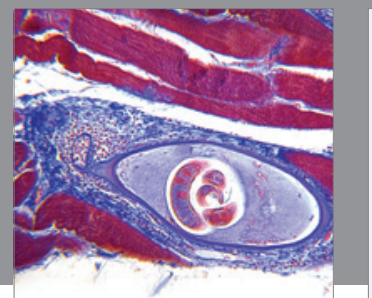

Gastroenterology

Research and Practice
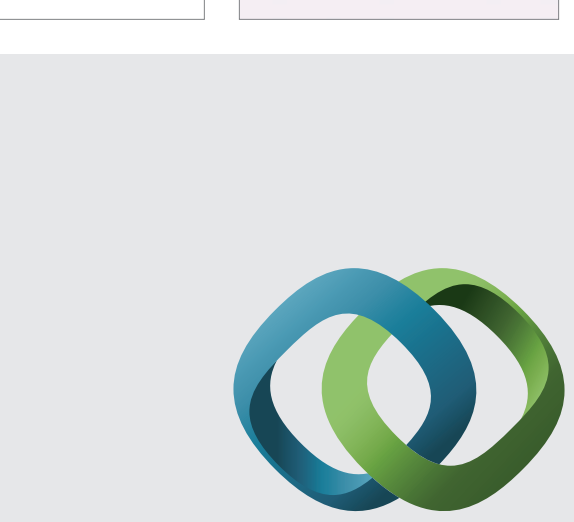

\section{Hindawi}

Submit your manuscripts at

http://www.hindawi.com
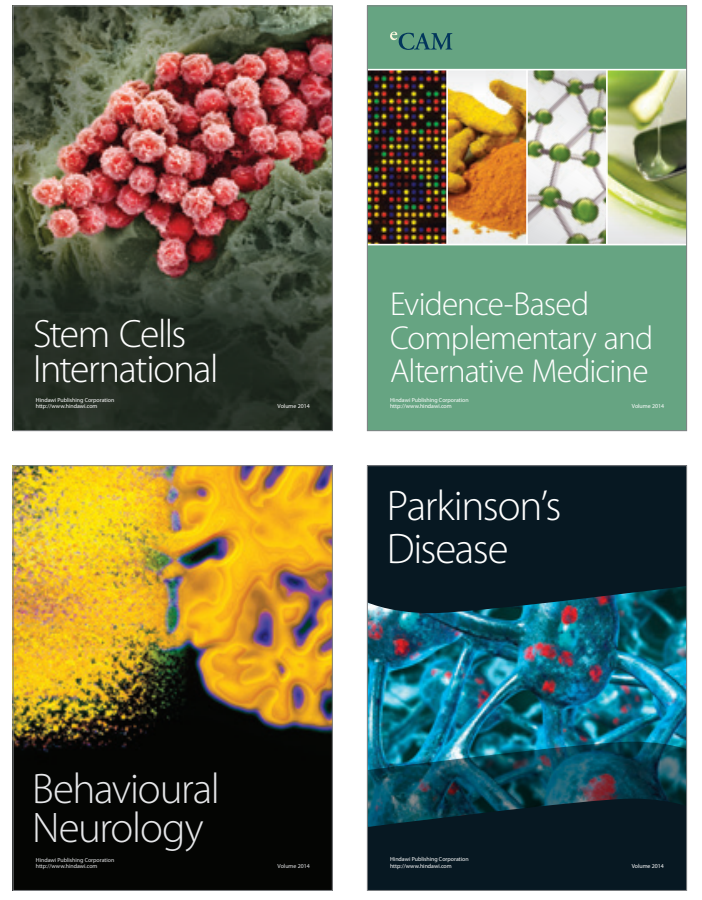
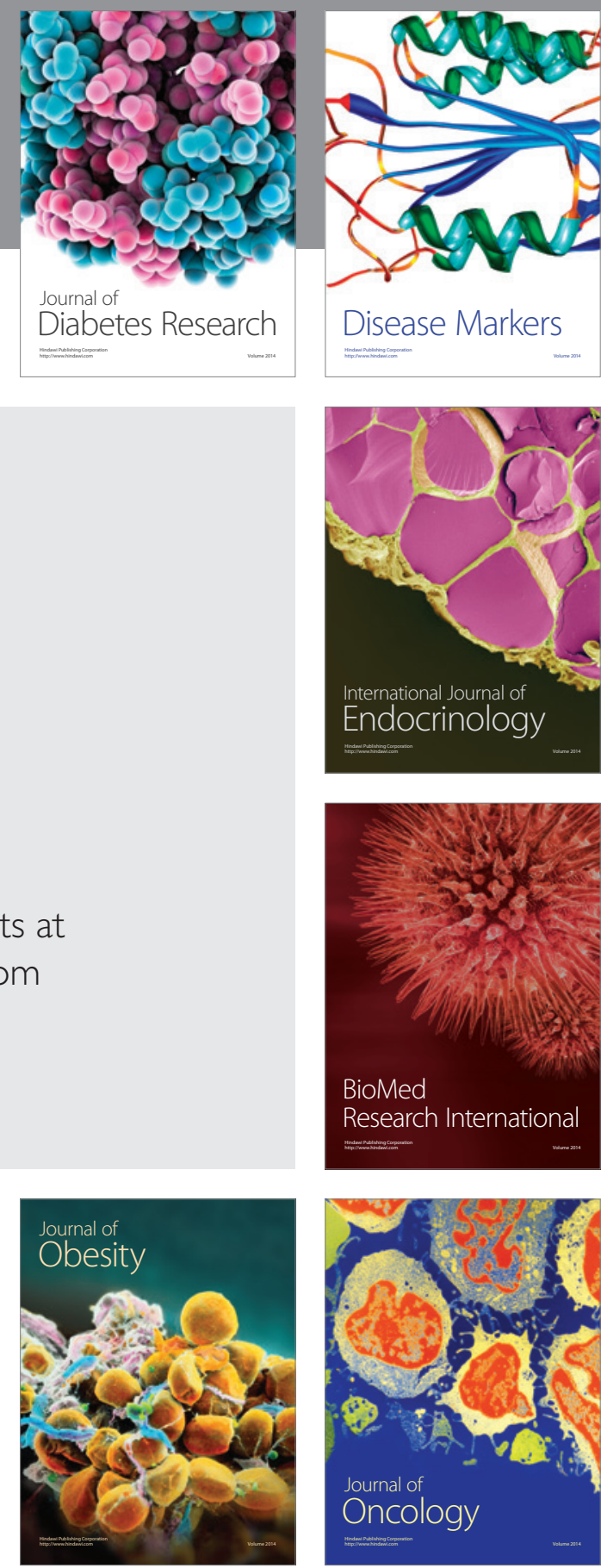

Disease Markers
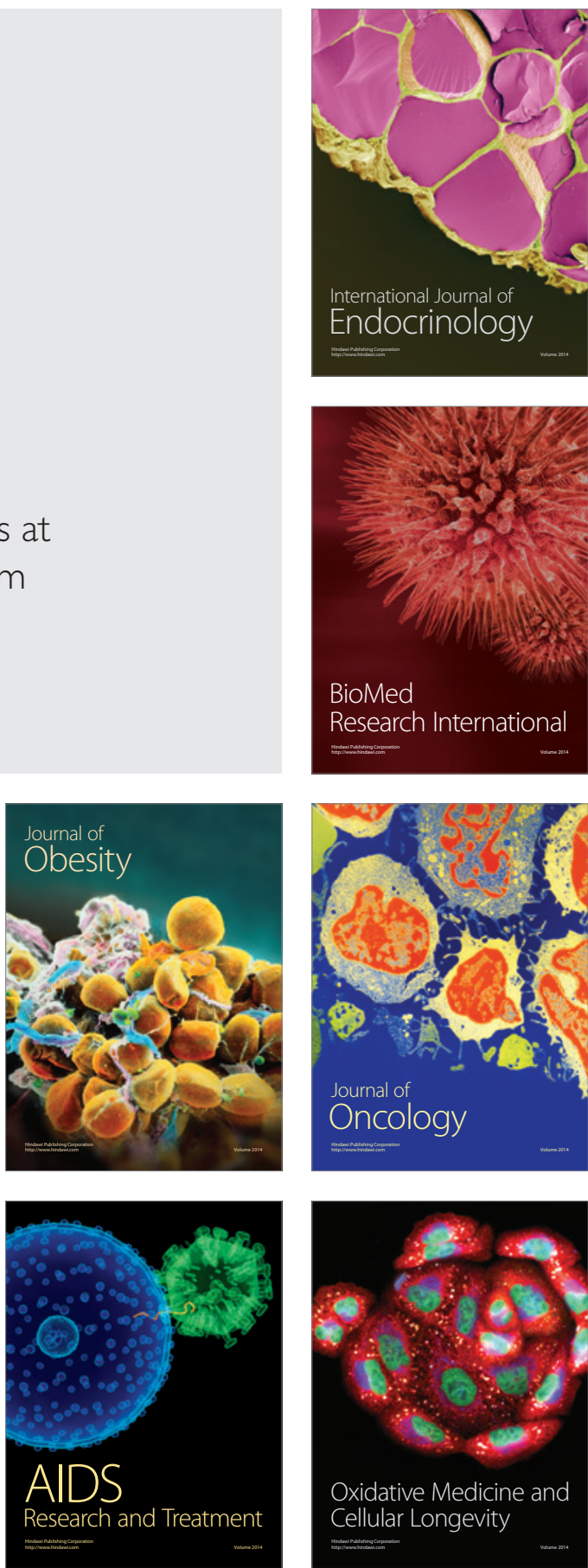